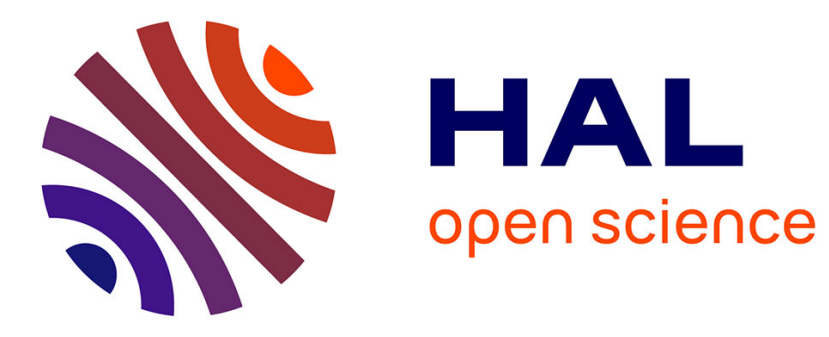

\title{
Normalization of T2W-MRI Prostate Images using Rician a priori
}

Guillaume Lemaître, Mojdeh Rastgoo, Joan Massich, Joan Vilanova, Paul

Walker, Jordi Freixenet, Anke Meyer-Baese, Fabrice Mériaudeau, Robert Martí

\section{To cite this version:}

Guillaume Lemaître, Mojdeh Rastgoo, Joan Massich, Joan Vilanova, Paul Walker, et al.. Normalization of T2W-MRI Prostate Images using Rician a priori. SPIE Medical Imaging, Feb 2016, San Diego, United States. hal-01265774

\section{HAL Id: hal-01265774 https://u-bourgogne.hal.science/hal-01265774}

Submitted on 5 Feb 2016

HAL is a multi-disciplinary open access archive for the deposit and dissemination of scientific research documents, whether they are published or not. The documents may come from teaching and research institutions in France or abroad, or from public or private research centers.
L'archive ouverte pluridisciplinaire HAL, est destinée au dépôt et à la diffusion de documents scientifiques de niveau recherche, publiés ou non, émanant des établissements d'enseignement et de recherche français ou étrangers, des laboratoires publics ou privés. 


\title{
Normalization of T2W-MRI Prostate Images using Rician a priori
}

\author{
Guillaume Lemaître $^{a, b}$, Mojdeh Rastgoo $^{a, b},{\text { Joan } \text { Massich }^{a} \text {, Joan C. Vilanova }}^{b}$, Paul M. Walker ${ }^{a}$, \\ Jordi Freixenet $^{b}$, Anke Meyer-Baese $^{c}$, Fabrice Mériaudeau $^{a}$, and Robert Martí ${ }^{b}$

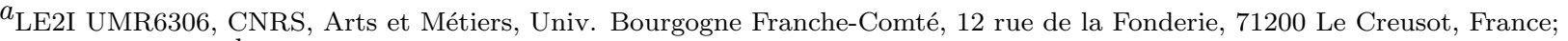

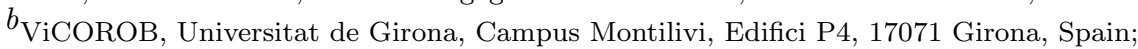 \\ ${ }^{c}$ Department of Scientific Computing, 400 Dirac Science Library, Florida State University, Tallahassee, FL 32306, US
}

\begin{abstract}
Prostate cancer is reported to be the second most frequently diagnosed cancer of men in the world. In practise, diagnosis can be affected by multiple factors which reduces the chance to detect the potential lesions. In the last decades, new imaging techniques mainly based on MRI are developed in conjunction with ComputerAided Diagnosis (CAD) systems to help radiologists for such diagnosis. CAD systems are usually designed as a sequential process consisting of four stages: pre-processing, segmentation, registration and classification. As a pre-processing, image normalization is a critical and important step of the chain in order to design a robust classifier and overcome the inter-patients intensity variations. However, little attention has been dedicated to the normalization of T2W-Magnetic Resonance Imaging (MRI) prostate images. In this paper, we propose two methods to normalize T2W-MRI prostate images: (i) based on a Rician a priori and (ii) based on a Square-Root Slope Function (SRSF) representation which does not make any assumption regarding the Probability Density Function (PDF) of the data. A comparison with the state-of-the-art methods is also provided. The normalization of the data is assessed by comparing the alignment of the patient PDFs in both qualitative and quantitative manners. In both evaluation, the normalization using Rician a priori outperforms the other state-of-the-art methods.
\end{abstract}

Keywords: Prostate cancer, T2W-MRI, normalization, pre-processing, computer-aided diagnosis

\section{INTRODUCTION}

Prostate Cancer $(\mathrm{CaP})$ has been reported the second most frequently diagnosed cancer of men accounting for $13.6 \% ! 1$ In United States, aside from skin cancer, CaP was considered to be the most commonly diagnosed cancer among men, implying that approximately 1 in 6 men will be diagnosed with CaP during their lifetime. The American cancer society also reported an estimated 233,000 new cases of prostate cancer in 2014! To address these dramatic issues, more systematic screenings are organized through Prostate-Specific Antigen (PSA) test with further Transrectal Ultrasound biopsy if necessary. However, these tests are unreliable or invasive and that is why further investigations using Magnetic Resonance Imaging (MRI)-Computer-Aided Diagnosis (CAD) are motivated. In the past decades, several CAD systems have been proposed in order to assist the radiologists with their diagnosis. These systems are usually designed as a sequential process consisting of four stages: preprocessing, segmentation, registration and classification. As a pre-processing steps, image normalization is an important step of the chain. Normalization is a highly crucial step to overcome the inter-patient intensity variations occurring, enforce the repeatability, and achieve a robust classification. ${ }^{[3}$ However, little attention has been dedicated to the problem of normalization of T2W-MRI prostate images. ${ }^{3}$

Artan et al $l^{45}$ and Ozer et al 6$]^{7}$ proposed to normalize the T2W-MRI images by computing the standard score (i.e., $z$-score) of the Peripheral Zone (PZ) pixels such as:

$$
I_{s}(x)=\frac{I_{r}(x)-\mu_{P Z}}{\sigma_{P Z}}, \forall x \in P Z,
$$

Further author information: (Send correspondence to G.L)

G.L.: E-mail: guillaume.lemaitre@udg.edu 


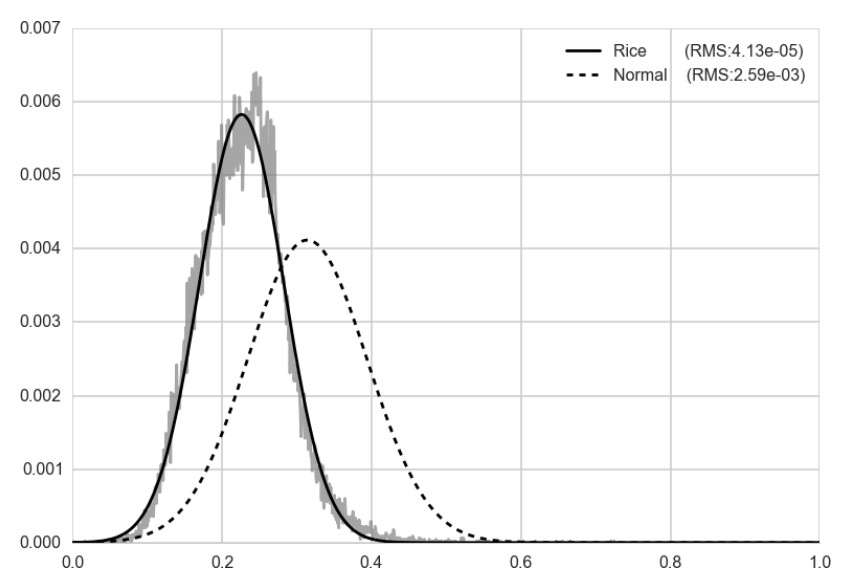

(a)

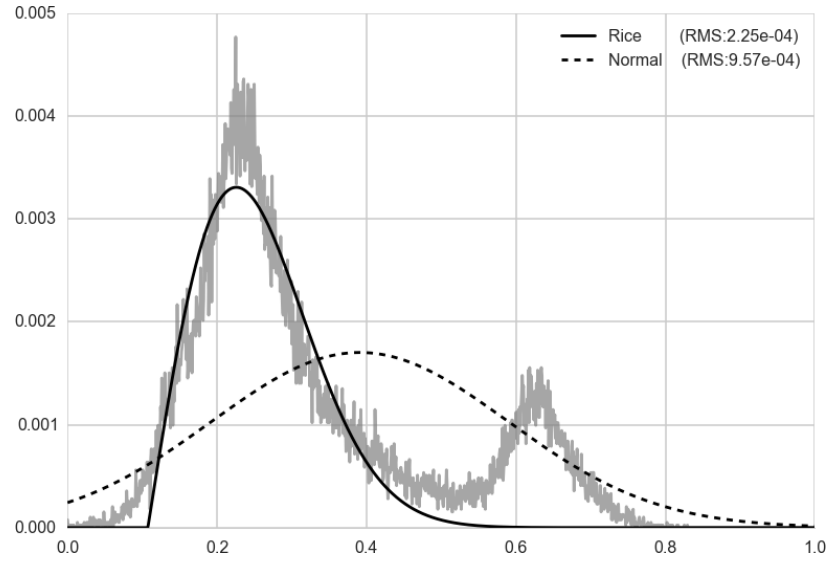

(b)

Figure 1: Visual evaluation of the goodness of fitting using Rician and Gaussian distribution.

where, $I_{s}(x)$ and $I_{r}(x)$ are the standardized and the raw signal intensity, respectively, and $\mu_{P Z}$ and $\sigma_{P Z}$ are the mean and standard deviation of the PZ signal intensity. This transformation enforces the image Probability Density Function (PDF) to have a zero mean and a unit standard deviation. However, this normalization is not appropriate if the PDF do not follow a Gaussian distribution as illustrated in Fig.1

Lv et al. ${ }^{8}$ used the method proposed by Nyul et al ${ }^{9}$ For a given patient, a warping function is inferred by matching some specific landmarks (i.e., median and different percentiles) of the current PDF to the same landmarks learned during a training phase from several patients. The mapping between each landmark is performed using a linear mapping. Viswanath et al ${ }^{10}$ used a variant of the previous method by segmenting first the image using region growing with a pre-defined homogeneity criterion and keeping only the largest region to build the PDF. Nevertheless, the warping functions inferred by these methods can suffer from abrupt changes around the landmarks position, leading to a disrupt PDF in the normalized image.

In this paper, we evaluate and compare different normalization approaches in the context of T2W-MRI prostate images normalization. Our contribution is threefold: (i) a normalization approach based on a Rician a priori; (ii) a normalization approach based on a method used in registration of functional data, without any assumption regarding the PDF of the data; (iii) a novel evaluation metric to asses quantitatively the alignment of the PDFs independently of the assumed distribution. These methods will be compared qualitatively and quantitatively, with both $z$-score normalization and piecewise-linear normalization.

\section{METHODOLOGY}

\subsection{Normalization using Rician a priori}

As stated in Sect.1. proper normalization of the MRI data during pre-processing is a key problem that has been addressed using parametric and non-parametric strategies. We believe that normalizing MRI data using a parametric model based on a Rician distribution would improve the results for the parametric case. Expecting this improvement by changing the data model from the widely used Gaussian distribution to Rician distribution is reasonable. Indeed, Bernstein et al ${ }^{11}$ state that MRI data theoretically follows a Rayleigh distribution for a low Signal-to-Noise Ratio (SNR) scenarios while it appears closer to a Gaussian distribution when the SNR increases. Figure 1 shows the intensity spectrum for some MRI prostate data as well as the fitted Gaussian and Rician distributions. A qualitative assessment of the underlying distribution is performed by overlying the fitted distribution, while quantitative results of the fitting are given in terms of Root Mean Square. It can be highlighted that the Rician model better fits the data than the Gaussian model.

The normalization is carried out as: (i) fit a Rician model to each prostate PDF using non-linear least squares minimization; (ii) compute the mean (see Eq. (2)) and variance (see Eq. (3)) of the Rician model; (iii) normalize 
the entire data using the $z$-score similarly as in Eq. (1).

$$
\begin{gathered}
\mu_{r}=\sigma \sqrt{\frac{\pi}{2}} L_{1 / 2}\left(-\frac{\nu^{2}}{2 \sigma^{2}}\right), \\
\sigma_{r}=2 \sigma^{2}+\nu^{2}-\frac{\pi \sigma^{2}}{2} L_{1 / 2}^{2}\left(\frac{-\nu^{2}}{2 \sigma^{2}}\right),
\end{gathered}
$$

where $\nu$ and $\sigma$ are the distance between the reference point and the center of the bivariate distribution and the scale, respectively; $L_{1 / 2}$ denotes a Laguerre polynomial.

\subsection{Normalization using generative models in functional data analysis}

Srivastava et al ${ }^{[12]}$ have proposed a generic method to register functional data, without any assumption regarding the models of different functions. This framework (see Sect.2.2.2 relies on the Square-Root Slope Function (SRSF) representation (see Sect. 2.2.1) which transforms the Fisher-Rao metric into the conventional $\mathbb{L}^{2}$ metric, and thus allows to define a cost function corresponding to an Euclidean distance between two functions in this new representation.

\subsubsection{Square-Root Slope Function representation}

In the proposed registration framework of functional data, two function $f_{1}$ and $f_{2}$ are registered by composing $f_{2}$ with a warping function $\gamma$ such that:

$$
\underset{\gamma \in \Gamma}{\arg \min } D_{F R}\left(f_{1},\left(f_{2} \circ \gamma\right)\right)
$$

where $D_{F R}$ is the Fisher-Rao distance and $\Gamma$ is the set of all the functions $\gamma$.

The SRSF representation is used to transform the functions and register them into this space. The SRSF of a function $f$ is defined as:

$$
q(t)=\operatorname{sign}(\dot{f}(t)) \sqrt{|\dot{f}(t)|},
$$

where $\dot{f}(t)$ corresponds to the derivative of $f$.

The major property of the SRSF representation used in the registration framework is the following: the composition of a function $f$ with a warping function $\gamma$ (i.e., $f \circ \gamma$ ) is equivalent to Eq. (6), using the SRSF representation.

$$
\tilde{q}(t)=(q(t) \circ \gamma) \sqrt{\dot{\gamma}}
$$

where $\dot{\gamma}$ is the derivative of $\gamma$.

Using this property, a cost function (named amplitude or $y$-distance) is defined to measure the similarity between two functions $f_{1}$ and $f_{2}$, expressed as in Eq. (7)

$$
D_{y}\left(f_{1}, f_{2}\right)=\inf _{\gamma \in \Gamma}\left\|q_{1}-\left(q_{2} \circ \gamma\right) \sqrt{\dot{\gamma}}\right\| .
$$




\subsubsection{Registration framework}

The registration framework consists into two steps. First, an initialization in which the Karcher mean $\mu_{f}$ is computed as in Eq. (8)

$$
\mu_{f}=\underset{f \in \mathcal{F}}{\arg \min } \sum_{i=1}^{n} D_{y}\left(f, f_{i}\right)^{2}
$$

Then, for each function $f_{i}$ : (i) compute $\gamma_{i}^{*}$ as in Eq. (9); (ii) compute $\tilde{q}_{i}$ as in Eq. (6); (iii) update $\mu_{f}$ as in Eq. (8) by replacing $f_{i}$ by $\tilde{f}_{i}$, using $\tilde{q}_{i}$.

$$
\gamma_{i}^{*}=\underset{\gamma \in \Gamma}{\arg \min } \sum_{i=1}^{n} D_{y}\left(\mu_{f}, f_{i}\right)^{2}
$$

where $n$ is the total number of functions to be aligned.

This step is iteratively performed based on the gradient of the cost function given in Eq. (8). We refer the reader to the work of Srivastava et al..$^{12}$ for more detailed discussion.

\section{EXPERIMENTS}

\subsection{Data}

The experiments are conducted on a subset of public multi-parametric MRI prostate publicly available dataset *13 $^{13}$ This dataset was acquired from a cohort of patients with higher-than-normal level of PSA. The acquisition was performed using a 3T whole body MRI scanner (Siemens Magnetom Trio TIM, Erlangen, Germany) using sequences to obtain T2W-MRI. Aside of the MRI examinations, these patients also underwent a guided-biopsy. Finally, the dataset was composed of a total of 20 patients of which 18 patients had biopsy proven CaP and 2 patients were "healthy" with negative biopsies. In this study, our subset consists of 17 patients with CaP. The prostate organ as well as the prostate zones (i.e., PZ, Central Gland) and CaP were manually segmented by an experienced radiologist.

\subsection{Implementation}

The different normalization methods are implemented in Python and publicly available in GitHulf The normalization based on SRSF uses the implementation ${ }^{\text {f }}$ of Tucker et al ${ }^{14}$

\subsection{Parameters}

The model fitting for the Gaussian and Rician normalization is performed as a non-linear least squares problem, using Levenberg-Marquardt optimization. The piecewise-linear normalization is performed using the following set of percentiles $s \in\{0,5,25,50,75,95,100\}$ as landmarks. In the SRSF-based normalization, the PDFs are smoothed using spline-based denoising method.

\section{RESULTS}

\subsection{Qualitative}

Figure 2 depicts the alignment of the different PDFs using the different methods implemented. All the methods seem to address the problem of the PDF alignment of the full prostate data. However, the Rician normalization seems to outperform the other methods when focusing solely on the CaP data. The PDF computed in this specific area is more skewed from its original shape in the case of the piecewise-linear normalization than with the three other normalization strategies. The SRSF normalization gets unstable due to the warping function $\gamma$ found which is in practise non-smooth. 

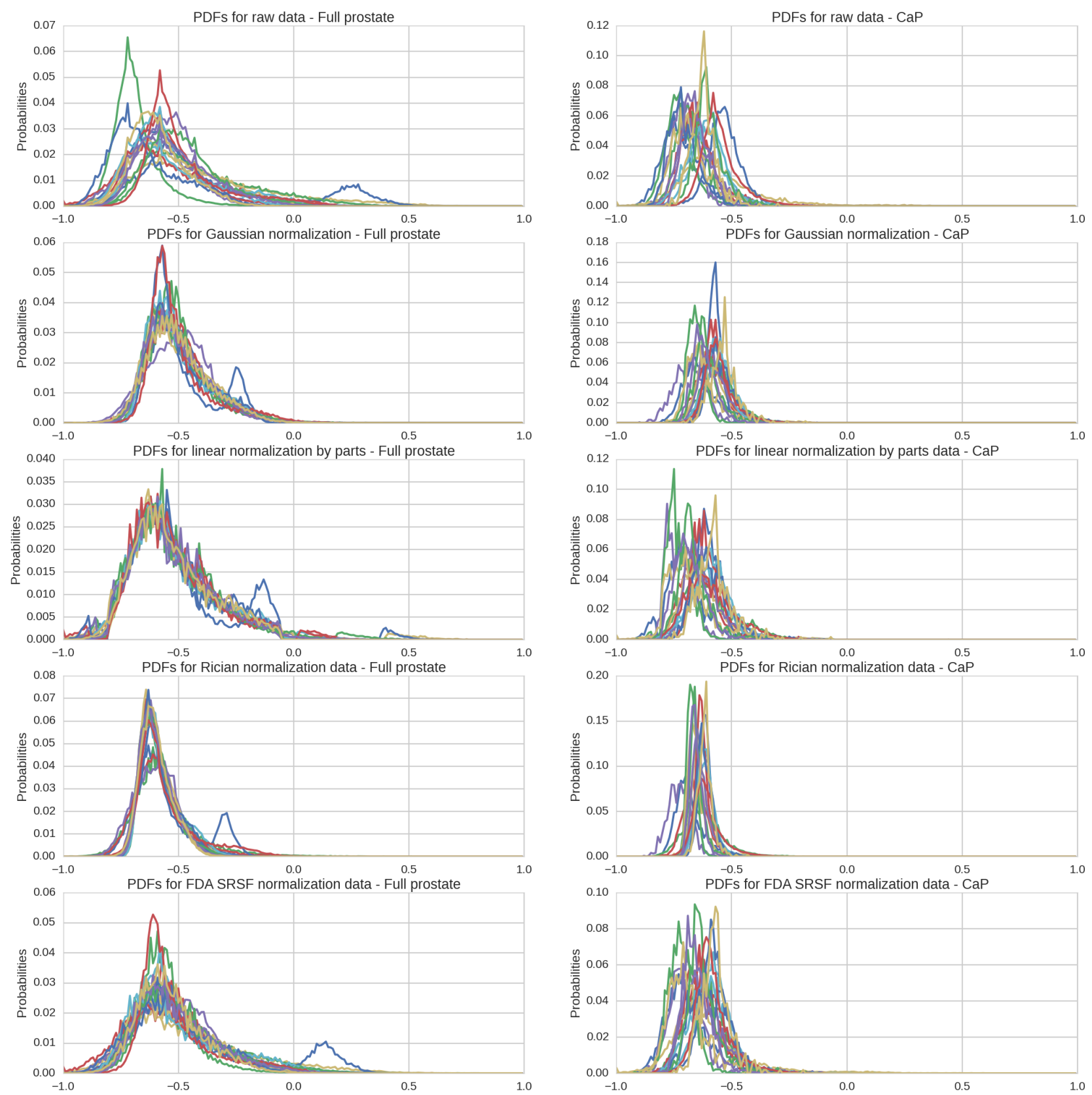

Figure 2: Qualitative evaluation by visual inspection of the alignment of the PDFs for the full prostate and the $\mathrm{CaP}$. 


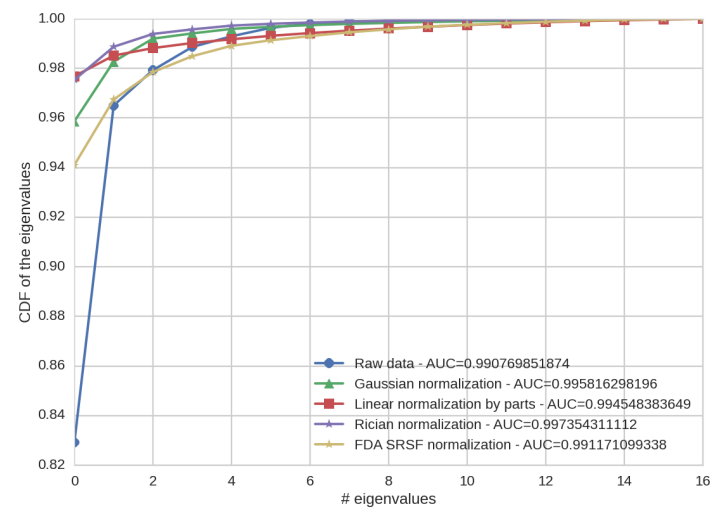

(a)

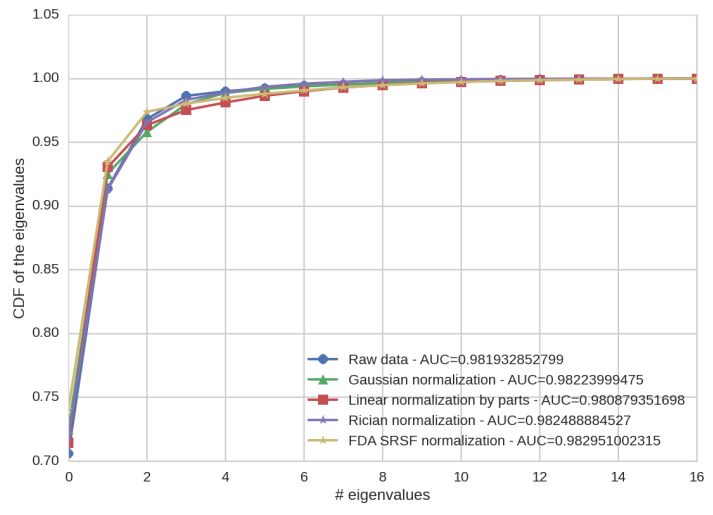

(b)

Figure 3: Spectral evaluation using PCA decomposition: (a) evaluation considering the full prostate, (b) evaluation considering only the $\mathrm{CaP}$.

\subsection{Quantitative}

A spectral evaluation is performed by decomposing the set of normalized PDFs using PCA under the assumption that they are linearly dependent. Intuitively, the eigenvalues of the PCA decomposition are correlated with the alignment of the different PDFs. Thus, in the case of a perfect alignment of the PDFs, the first eigenvalue is much greater than the remaining since that the first eigenvector encodes all the information. In the contrary, in the case of a misalignment of the PDFs, more eigenvectors are needed to encode the information synonymous with larger eigenvalues. Thus, we propose to use the cumulative sum of the normalized eigenvalues as well as the Area Under this Curve (AUC), as depicted in Fig. 3. Rician normalization outperforms the other methods with an AUC of 0.9974 and 0.9824 considering the full prostate and $\mathrm{CaP}$, respectively.

\section{CONCLUSION AND FUTURE WORK}

In this paper, we propose to normalize the T2W-MRI prostate images using two new strategies: (i) based on a Rician a priori and (ii) based on a SRSF representation which do not make any assumption regarding the PDF of the data. An extensive comparison was conducted showing that the Rician normalization outperforms the Gaussian, SRSF-based, and piecewise-linear normalization for T2W-MRI prostate images normalization.

As avenues for future research, the contribution of the Rician normalization must be evaluated in a classification framework. Furthermore, normalized T2W-MRI can be included with other modalities in order to perform classification using multi-parametric MRI data.

\section{Acknowledgments}

G. Lemaitre was supported by the Generalitat de Catalunya (grant nb. FI-DGR2012), the Spanish R+D+I grant TIN2012-37171-C02-01, and the Regional Council of Burgundy (grant nb. 2015-9201AAO050S02760). Calculations were performed using HPC resources from DSI-CCUB (Université de Bourgogne).

\section{REFERENCES}

1. J. Ferlay, H. R. Shin, F. Bray, D. Forman, C. Mathers, and D. M. Parkin, "Estimates of worldwide burden of cancer in 2008: GLOBOCAN 2008," International Journal of Cancer 127(12), pp. 2893-2917, 2010.

\footnotetext{
*http://visor.udg.edu/i2cvb/

https://github.com/glemaitre/protoclass

https://bitbucket.org/tetonedge/fdasrsf
} 
2. A. C. Society, "Cancer facts \& figures 2014," 2014.

3. G. Lemaitre, R. Marti, J. Freixenet, J. C. Vilanova, P. M. Walker, and F. Meriaudeau, "Computer-aided detection and diagnosis for prostate cancer based on mono and multi-parametric mri: A review," Computers in Biology and Medicine 60, pp. 8-31, 2015.

4. Y. Artan, M. A. Haider, D. L. Langer, T. H. van der Kwast, A. J. Evans, Y. Yang, M. N. Wernick, J. Trachtenberg, and I. S. Yetik, "Prostate cancer localization with multispectral mri using cost-sensitive support vector machines and conditional random fields," Image Processing, IEEE Transacrions on 19(9), pp. 2444-2455, 2010.

5. Y. Artan, D. Langer, M. Haider, T. van der Kwast, A. Evans, M. Wernick, and I. Yetik, "Prostate cancer segmentation with multispectral mri using cost-sensitive conditional random fields," in Biomedical Imaging: From Nano to Macro, 2009. ISBI '09. IEEE International Symposium on, pp. 278-281, 2009.

6. S. Ozer, M. Haider, D. Langer, T. van der Kwast, A. Evans, M. Wernick, J. Trachtenberg, and I. Yetik, "Prostate cancer localization with multispectral mri based on relevance vector machines," in Biomedical Imaging: From Nano to Macro, 2009. ISBI'09. IEEE International Symposium on, pp. 73-76, 2009.

7. S. Ozer, D. L. Langer, X. Liu, M. A. Haider, T. H. van der Kwast, A. J. Evans, Y. Yang, M. N. Wernick, and I. S. Yetik, "Supervised and unsupervised methods for prostate cancer segmentation with multispectral mri," Medical physics 37(4), pp. 1873-1883, 2010.

8. D. Lv, X. Guo, X. Wang, J. Zhang, and J. Fang, "Computerized characterization of prostate cancer by fractal analysis in mr images," Journal of Magnetic Resonance Imaging 30(1), pp. 161-168, 2009.

9. L. G. Nyul, J. K. Udupa, and X. Zhang, "New variants of a method of mri scale standardization," Medical Imaging, IEEE Transacrions on 19(2), pp. 143-150, 2000.

10. S. E. Viswanath, N. B. Bloch, J. C. Chappelow, R. Toth, N. M. Rofsky, E. M. Genega, R. E. Lenkinski, and A. Madabhushi, "Central gland and peripheral zone prostate tumors have significantly different quantitative imaging signatures on 3 tesla endorectal, in vivo t2-weighted mr imagery," Journal of Magnetic Resonance Imaging 36(1), pp. 213-224, 2012.

11. M. A. Bernstein, D. M. Thomasson, and W. H. Perman, "Improved detectability in low signal-to-noise ratio magnetic resonance images by means of a phase-corrected real reconstruction," Medical Physics 16(5), pp. 813-817, 1989.

12. A. Srivastava, E. Klassen, S. Joshi, and I. Jermyn, "Shape analysis of elastic curves in euclidean spaces," Pattern Analysis and Machine Intelligence, IEEE Transactions on 33, pp. 1415-1428, July 2011.

13. G. Lemaitre, J. Massich, R. Marti, J. Freixenet, J. C. Vilanova, P. M. Walker, D. Sidibe, and F. Meriaudeau, "A boosting approach for prostate cancer detection using multi-parametric mri," in The International Conference on Quality Control by Artificial Vision 2015, pp. 95340A-95340A, International Society for Optics and Photonics, 2015.

14. J. D. Tucker, W. Wu, and A. Srivastava, "Generative models for functional data using phase and amplitude separation," Computational Statistics \& Data Analysis 61, pp. 50-66, 2013. 